Revista de Ciencias Sociales - Número 65 (2014) - Páginas $185-209$

Evaluación de la didáctica y la satisfacción de los estudiantes...

\title{
EVALUACIÓN DE LA DIDÁCTICA Y LA SATISFACCIÓN DE LOS ESTUDIANTES EN UNA ASIGNATURA DE HISTORIA DEL DERECHO
}

\author{
EVALUATION OF TEACHING AND SATISFACTION \\ OF STUDENTS IN A COURSE OF HISTORY OF LAW
}

\author{
JUAN PABLO ZAMBRANO TIZNADO* \\ Universidad de la Frontera, Chile \\ juanpablo.zambrano@ufrontera.cl \\ ANDRÉS SÁEZ GEOFFROY** \\ Universidad de la Frontera, Chile \\ andres.saez@ufrontera.cl \\ GUILLERMO ZAMBRANO TIZNADO*** \\ Universidad de la Frontera, Chile \\ g.zambrano01@ufromail.cl
}

\section{Resumen}

El objetivo de este trabajo es presentar los resultados de una investigación llevada a cabo en la asignatura de Historia del Derecho correspondiente al primer año de la carrera de Licenciatura en Ciencias

* Académico del Departamento de Ciencias Jurídicas. Doctorando en Ciencias Humanas (Universidad Austral de Chile). Becario CONICYT de Postgrado Nacional. Artículo recibido el 3 de junio de 2014 y aceptado el 27 de julio de 2014.

* Académico del Departamento de Ciencias Sociales. Doctorando en Historia (Universitat de Barcelona, España), Magíster en Estudios Históricos Avanzados (Universitat de Barcelona, España).

** Estudiante de Magíster en Gerencia Social (Universidad de la Frontera (Chile), Postitulado en Educación Tecnológica (Universidad del Pacífico, Chile).

Revista de Ciencias Sociales - Número 65 (2014) - Universidad de Valparáíso - ISSN 0716-7725-Valparáiso, Chile 
Jurídicas. Se trata de una investigación exploratoria que vincula la didáctica de la historia con la satisfacción de los estudiantes. Se concluye que existen importantes dificultades en la implementación didáctica de la asignatura, además de insatisfacción de los estudiantes, mala utilización de herramientas tics y la no utilización de fuentes primarias.

\section{Palabras claves}

Historia del derecho, aprendizaje de la historia, discurso pedagógico.

\section{Abstract}

The aim of this paper is to present the results of a research carried out in the course of history for the first year of the Bachelor of Science in Legal law. This is an exploratory research linking historical education with student satisfaction. It is concluded that there are significant difficulties in implementing the teaching of the subject, in addition to student dissatisfaction, misuse of tools tics and non-use of primary sources.

\section{Keywords}

History of law, learning history, teaching speech.

\section{Introducción}

El objetivo de este trabajo es presentar los resultados de una investigación llevada a cabo en la asignatura de Historia del Derecho correspondiente al primer año de la carrera de Licenciatura en Ciencias Jurídicas. Se trata de una investigación diagnóstica que se enmarca dentro de una investigación mayor que pretende utilizar los resultados que se presentan como insumos que permitan diseñar una transposición didáctica en un curso de Historia del Derecho. En concreto, esta investigación se concentra en la correlación entre el uso de estrategias didácticas y la satisfacción de los estudiantes. Se ha elegido esta asignatura porque aparentemente la enseñanza de la historia en los distintos niveles educativos de Chile, ha estado tradicionalmente vinculada a un carácter enciclopédico y memorístico. Por cierto, tanto la excesiva memorización como el carácter enciclopédico parecen ser aspectos comunes de los estudios jurídicos en Chile ${ }^{1}$.

1. El Dr. Coloma utiliza la figura de un personaje literario (el profesor Binns, de la saga Harry Potter), como hilo conductor para mostrar el estado actual de

Facultad de Derecho y Ciencias Sociales - Universidad de Valparaíso - Chile 
Las carreras de Derecho no son inmunes a los problemas comunes de la educación superior en Chile. En este sentido, una de las grandes críticas a la docencia universitaria se refiere a la ausencia de conocimientos y herramientas pedagógicas en el grueso de los profesores que dictan cátedras en los cursos de programas de pregrado y postgrado no ligados a las Ciencias de la Educación. Dicha situación ha tratado de remediarse mediante la implementación de diversos programas y diplomados de nivelación de competencias docentes tanto para académicos en ejercicio como para aquellos que se están integrando (y carecen obviamente de formación inicial en el área pedagógica) al mundo universitario. A modo de ejemplo, programas como el Diplomado en Docencia de Educación Superior de la Pontificia Universidad Católica de Valparaíso, el Portal de Desarrollo Docente de las Universidades Andrés Bello y de las Américas (en modalidad online), el programa Moodle de la Universidad de Playa Ancha, etc. No obstante, la mayor parte de las Universidades no han implementado estas modalidades de nivelación. Además, en Chile, y pese a la evidente importancia que tiene la profesión de abogado, no existen reportes que entreguen información más allá de algunos rankings sobre la calidad del pregrado en Derecho ${ }^{2}$, tampoco existen estudios amplios y empíricos sobre educación legal ni insumos que permitan evaluar la calidad de la formación de los futuros abogados. Entonces, este trabajo es un diagnóstico de la situación actual en base a los resultados de los estudiantes en un curso de Historia General del Derecho y de las percepciones de estos en torno a los aprendizajes que han logrado y las innovaciones didácticas que consideran más pertinentes.

los currículos, metodologías de la enseñanza y evaluación de aprendizajes de las Ciencias Jurídicas en Chile. No parece casual que el personaje elegido sea el docente de Historia de la Magia en la Academia Hogwarts. COLOMA, Rodrigo: "El ocaso del profesor Binns. Un ensayo acerca de la enseñanza del derecho en Chile”. En: Ius et Praxis. Talca, Chile. 2005, Vol.11. No 1. Págs. 133-172.

2. En otra oportunidad he sostenido la relación entre información pública y educación de calidad. cfr. AGÜERO, Claudio, y ZAMBRANO, Juan Pablo: "El derecho de acceso a la información como garantía de una educación de calidad". En: Revista de Ciencias Sociales, Universidad de Valparaíso, Facultad de Derecho. Valparaíso, Chile. 2009, N 54. Págs. 133-147.

Revista de Ciencias Sociales - Número 65 (2014) - Universidad de Valparáíso - ISSN 0716-7725-Valparáiso, Chile 
En cuanto al currículo de la carrera de Derecho, este no ha sufrido históricamente modificaciones de importancia ${ }^{3}$. En particular, para el caso de la Historia del Derecho, y pese a las diversas denominaciones de la asignatura ('Aspectos Históricos del Derecho', 'Derecho Histórico', 'Historia del Derecho' e 'Historia del Derecho Chileno') sus contenidos en general han permanecido inalterados. En otro sentido, y siendo evidente el carácter interdisciplinario de la Historia del Derecho, en Chile existen muy pocos profesores de Historia del Derecho que son historiadores profesionales. El problema es mayor si se considera que no existen iushistoriadores chilenos que hayan reflexionado teóricamente sobre la naturaleza de su disciplina y tampoco los teóricos del derecho dedicados a la teoría de la ciencia jurídica, han desarrollado dicha labor.

\section{Objetivos de la Investigación}

- Identificar las falencias que presenta en el aprendizaje de los estudiantes el uso de una metodología tradicional de enseñanza en la asignatura Historia General del Derecho.

- Determinar las expectativas que los alumnos manifiestan respecto a un cambio en la didáctica de la asignatura.

\section{Marco teórico}

La globalización trae consigo grandes cambios en la tecnología, las ciencias y el mundo laboral, imponiendo nuevos desafíos a la educación legal con miras a lograr en el proceso de enseñanzaaprendizaje las aptitudes y actitudes necesarias para un correcto ejercicio profesional. Por otro lado, los cambios tecnológicos acelerados así como la mejora de los instrumentos electromagnéticos, la biología, la física, en la economía el neoliberalismo y los tratados de libre comercio han generado que "el conocimiento (de base disciplinaria, publicado y

3. SOLARI, Enzo: "El currículo chileno de estudios jurídicos". En: Revista de Derecho de la Pontificia Universidad Católica de Valparaíso. Valparaíso, N $^{\circ} 39$, 2012. Págs. 703-734.

Facultad de Derecho y Ciencias Sociales - Universidad de Valparaíso - Chile 
registrado internacionalmente) [que demoró] 1750 años en duplicarse por primera vez en la era cristiana, $[\ldots]$, se estima que hacia el año 2020 se duplicará cada 73 días" ${ }^{2}$. Estos cambios implican nuevos requerimientos profesionales, además del trabajo interdisciplinario: "[s] ea como fuere, resulta evidente que la educación enfrenta en este ámbito un acelerado cambio de contexto, desde el momento que contingentes cada vez más amplios se emplean en el sector servicios donde una de las exigencias es haber adquirido las necesarias destrezas interpersonales" 5 . Se trata entonces, de adaptar la educación universitaria de tipo enciclopedista y verbal a los nuevos cambios asociados a la llamada sociedad del conocimiento y la información.

De esta manera, organismos como la OCDE y la UNESCO, hacen un llamado de atención respecto a lo mutable de lo cognoscible, donde el ser humano debe estar constantemente aprendiendo y mejorando su accionar. De este modo, se asume en la actualidad que la educación posee dos dimensiones, por un lado entregar cada día un mayor volumen de contenidos adaptados para el correcto desarrollo humano y por otro, orientar esos conocimientos para proyectarlos en la vida en sociedad. En términos de lo expuesto en el informe Delors "ya no basta con que cada individuo acumule al comienzo de su vida una reserva de conocimientos a la que podrá recurrir después sin límites. Sobre todo, debe estar en condiciones de aprovechar y utilizar durante toda la vida cada oportunidad que se le presente de actualizar, profundizar y enriquecer ese primer saber y de adaptarse a un mundo en permanente cambio"6.

Entonces, para lograr unir los aprendizajes se necesita que los sistemas educativos desarrollen ciertos mecanismos que sean capaces

4. BRUNNER, José Joaquín: “Globalización y el futuro de la educación: tendencias, desafíos y estrategias”. En: Seminario sobre prospectiva de la educación en la región de América Latina y el Caribe, UNESCO Santiago, Chile. 23 al 25 de agosto del 2000. Pág. 8.

5. Ídem.

6. DELORS, Jaques: La educación encierra un tesoro, Editorial Santillana, UNESCO, 1996. Pág. 95.

Revista de Ciencias Sociales - Número 65 (2014) - Universidad de Valparáíso - ISSN 0716-7725-Valparaíso, Chile 
de desplegar no sólo aprendizaje de conocimientos, sino también capacidades y destrezas que se puedan aplicar en otros contextos, adaptables a las diversas condiciones del medio y que puedan favorecer una enseñanza y aprendizaje que integre a todos los actores sociales. Se trata de evitar que "los sistemas educativos formales propendan a dar prioridad a la adquisición de conocimientos, en detrimento de otras formas de aprendizaje, importa concebir la educación como un todo"7. Asumiendo que esta investigación pretende sentar los lineamientos para una futura trasposición didáctica, nos ha parecido necesario explicitar los criterios pedagógicos sobre los cuales se sustentará la futura propuesta didáctica. Hemos elegido la teoría cognoscitiva y el modelo de aprendizaje denominado constructivismo, cuyo ideólogo central es Jean Piaget. En breves palabras podemos definir el constructivismo, como un proceso donde "la enseñanza corre a cargo del enseñante como su originador; pero al fin y al cabo es una construcción conjunta como producto de los continuos y complejos intercambios con los alumnos y el contexto instruccional (institucional, cultural, etcétera), que a veces toma caminos no necesariamente predefinidos en la planificación" En este sentido, el constructivismo tendría dos elementos esenciales: el primero, es la construcción del saber por parte del docente a partir de su propio bagaje cultural o intelectual. El segundo, es que el centro del proceso del aprendizaje es el educando y no el enseñante implicando un cambio en el paradigma de la enseñanza tradicional la que se encuentra, según Habermas ${ }^{9}$ centrada en lo técnico-conceptual y con un fuerte carácter descendente a partir del docente ${ }^{10}$. Bajo el paradigma anterior, Piaget señala que en la infancia se da importancia a la

7. Ibídem. Pág. 54.

8. DÍAZ, Frida. y HERNÁDEZ Gerardo: Estrategias Docentes para un Aprendizaje Significativo: una interpretación constructivista. México: Mc Graw Hill, 2002. pág. 143, Vid. EGGEN Paul D. KAUCHAK Donald P.: Estrategias docentes: Enseñanza de contenidos curriculares y desarrollo de habilidades de pensamiento. D.F, México. Editorial Fondo de cultura económica, 2005.

9. Cfr. HABERMAS Jürgen: Ciencia y técnica como ideología. Madrid, España. Editorial Tecnos, 1986.

10. Cfr. GRUNDY Shirley: Producto o praxis del currículum, Madrid, España. Editorial Morata, 1999.

Facultad de Derecho y Ciencias Sociales - Universidad de Valparaíso - Chile 
organización y adaptación de diversos conocimientos que hacen que el niño se integre a las estructuras sociales que le rodean predominando el pensamiento concreto, pero una vez que ha alcanzado la adolescencia, sus estructuras mentales cambian y se vuelven abstractas. En esta transformación "las funciones esenciales de la inteligencia consisten en comprender e inventar. Dicho de otra manera: en construir estructuras, estructurando lo real" ${ }^{11}$. De esta manera, en los procesos de asimilación y acomodación de conocimientos que realiza un individuo (aprendizajes) a nivel cognitivo, el rol del docente radica en transformarse en el facilitador de las experiencias educativas y de autoconstrucción cognitiva de los estudiantes, al mismo tiempo que debe evitar asumirse como el centro transmisor del saber. Junto a lo anterior, se hace necesario centrar el proceso en la llamada enseñanza activa y en el rol del profesor estratégico. Siguiendo a Pérez Cabaní1 ${ }^{12}$ este paradigma sostiene que el docente es un experto en su especialidad:

[...] siendo capaz de planificar y organizar tareas de clase, de relacionar la nueva materia con los temas trabajados anteriormente; es un mediador que guía el pensamiento de los alumnos hacia metas apropiadas que permiten a sus estudiantes asumir, progresivamente, la responsabilidad de su propio proceso de aprendizaje; es capaz de enseñar a construir la propia auto imagen cognitiva, es decir, a identificar las propias habilidades, preferencias y dificultades al momento de aprender; insiste en la reflexión sobre los procesos de pensamiento seguidos por los alumnos para resolver problemas en el aula; proporciona procedimientos de trabajo y de investigación basados en cuestiones como dónde y cómo buscar seleccionar información relevante, y establece sistemas de evaluación que permiten la reelaboración de las ideas enseñadas y concibe la evaluación como una oportunidad de que el alumno aplique de manera autónoma los procedimientos de aprendizaje $\mathrm{e}^{13}$.

11. Cfr. PIAGET, Jean, Psicología y pedagogía. Barcelona, España. Editorial Ariel, 2001.

12. PÉREZ CABANÍ, María: "La formación del profesorado para enseñar estrategias de aprendizaje”. En: Moreno, Carlos, Estrategias de enseñanza y aprendizaje: formación del profesorado y aplicación en la escuela. Editorial Visor, 2002. Pág. 30.

13. Para profundizar en el enfoque de la enseñanza activa véase el texto de EggenKauchak, ob. cit. Págs. 20-87.

Revista de Ciencias Sociales - Número 65 (2014) - Universidad de Valparáíso - ISSN 0716-7725-Valparaíso, Chile 
Bajo este prisma, un académico debiera ser capaz de realizar un cambio en su práctica pedagógica asumiéndose como protagonista en el aprendizaje significativo de sus estudiantes. Cuando un profesor está trabajando como un profesor técnico, tiene mucho que hacer para poder producir un cambio en su práctica hasta lograr ser un profesor crítico y estratégico $^{14}$. En definitiva, si relacionamos el rol docente con la teoría constructivista debemos comprender que la práctica docente se debe orientar a la enseñanza de las herramientas necesarias para que los estudiantes puedan construir nuevos conocimientos.

Como resulta obvio, es necesario que el profesor utilice los conocimientos previos del estudiante posibilitando la potenciación de habilidades para descubrir y generar conocimientos; en este sentido, resulta preferible que el docente se enfoque en los aspectos importantes y novedosos de los temas y actividades que generan nuevos conocimientos para conocer, hacer y ser, con el fin de generar aprendizajes significativos y de largo plazo. La teoría del aprendizaje significativo planteada por David Ausubel "se distingue por dos características, la primera es que su contenido puede relacionarse de un modo sustantivo, no arbitrario o al pie de la letra, con los conocimientos previos del alumno, y la segunda es que éste ha de adoptar una actitud favorable para tal tarea, dotando de significado propio a los contenidos que asimila"15. A partir de estas características se le define como "el proceso según el cual se relaciona un nuevo conocimiento o información con la estructura cognitiva del que aprende de forma no arbitraria y sustantiva o no literal. Esa interacción con la estructura cognitiva no se produce considerándola como un todo, sino con aspectos relevantes presentes en la misma, que reciben el nombre de subsumidores o ideas de anclaje" ${ }^{16}$. Esta teoría se contrapone al clásico aprendizaje memorístico

14. HABERMAS, ob. cit.

15. COOL, César: "Aprendizaje por descubrimiento frente a aprendizaje por recepción”. En: Juan García Madruga. Desarrollo psicológico y educación. Madrid, España. Editorial Alianza. 1999.

16. RODRÍGUEZ, Luz: La teoría del aprendizaje significativo. Tenerife, España. Centro de educación a distancia, 2008. Pág. 11.

Facultad de Derecho y Ciencias Sociales - Universidad de Valparaíso - Chile 
y señala que los nuevos aprendizajes deben generarse a partir de los conocimientos que ya posee el alumno, forjando mayores redes de relación entre lo conocido y lo por conocer. El aprendizaje se produce activamente a través de la relación de conocimientos previos y presentes, seleccionando y construyendo estructuras, mapas conceptuales y mentales. En este sentido, docente y educando deben generar un diálogo donde la información es trabajada y elaborada por el alumno guiado por el profesor.

La teoría del aprendizaje significativo propone que "1) Los nuevos materiales que van a ser aprendidos deben ser potencialmente significativos; es decir, suficientemente sustantivos y no arbitrarios para poder ser relacionadas con las ideas relevantes que posea el sujeto. 2) La estructura cognoscitiva previa del sujeto debe poseer las necesarias ideas relevantes para que puedan ser relacionadas con los nuevos conocimientos. 3) El sujeto debe manifestar una disposición significativa hacia el aprendizaje" ${ }^{17}$. Por lo anterior, se hace necesario que las actividades que se planifiquen por el docente tengan elementos de innovación dejando de lado lo memorístico. Entonces, el docente debe conocer qué tipo de información posee el estudiante y deben existir entes motivacionales dirigidos desde el profesor con el fin de invitar a que el estudiante demuestre interés en la actividad planteada.

Esta noción de descubrimiento del aprendizaje, conlleva que "los métodos de descubrimiento guiados implican proporcionar a los estudiantes oportunidades para manipular objetos en forma activa y transformarlos por medio de la acción directa, así como actividades que los animen a buscar, explorar, analizar o procesar de alguna otra manera la información que reciben en lugar de sólo responder a ella. En teoría, estas oportunidades no sólo incrementan el conocimiento de los estudiantes acerca del tema que tienen a la mano sino que estimulan su curiosidad y los ayudan a desarrollar estrategias generalizadas para aprender a aprender útiles para descubrir conocimiento en otras situaciones"18. La idea es que el estudiante explore y descubra — valga

17. COLL, ob. cit. Pág. 84.

18. GOOG, Thomas y BROPHY, Jere E.: Psicología educativa contemporánea. D.F., México. Editorial McGraw Hill, 2000. Pág. 1.

Revista de Ciencias Sociales - Número 65 (2014) - Universidad de Valparáíso - ISSN 0716-7725-Valparaíso, Chile 
la redundancia - nuevos conocimientos es decir, el profesor no entrega el conocimiento como producto elaborado sino que los conceptos son elaborados por los mismos estudiantes guiados por el profesor a partir de la elaboración de hipótesis y simulaciones. En este sentido se requeriría:

- La manipulación de objetos por parte del estudiante para que pueda transformarlos y usarlos acorde a las necesidades de aprendizaje y lo que se les pida realizar en una actividad específica.

- Necesidad de actividades que les animen a buscar e indagar elementos que no conocen y les puedan resultar novedosos de modo que puedan manipular la información a la cual tienen acceso y no sólo ser entes que responden a la información entregada.

- Necesidad de que los estudiantes tengan acción directa sobre la información y puedan incidir en los resultados que obtienen.

- Como es el estudiante el participe, lógicamente ocurrirán errores pero los errores aquí no pueden ser visto como algo negativo sino como la necesidad de redescubrir nuevamente y evitar volver a cometerlos.

Ahora bien, comprendiendo de mejor manera la forma de aprendizaje de los estudiantes, se puede abordar una investigación en Didáctica, la que abarca por un lado las estrategias de enseñanza de una determinada disciplina y por otro la investigación referida a la formación de profesores en cuanto estos se insertan en determinados contextos de enseñanza y cómo se desenvuelven a partir de la formación inicial docente ${ }^{19}$. Como señala Prats "La investigación en didáctica de

19. HENRÍQUEZ, Rodrigo y PAGÉS, Joan: "La investigación en didáctica de la historia”. En: Educación XXI Revista de la Facultad de Educación de la Universidad Autónoma de Barcelona, España. 2004, No 7, págs. 63-84. PAGÉS, Joan, "Enseñar a enseñar Historia: la formación didáctica de los futuros profesores de Historia”. En: Llopis, Miguel. Miradas a la historia. Murcia: Universidad de Murcia. 2004. Págs. 155-177.

Facultad de Derecho y Ciencias Sociales - Universidad de Valparaíso - Chile 
las ciencias sociales debe considerarse, en primer lugar, como un campo de investigación educativa, situado en la encrucijada de diversas disciplinas sociales entre las que están, por un lado, las que se ocupan tradicionalmente del aprendizaje y la enseñanza y, por otro, las que constituyen la base de los conocimientos que se pretenden enseñar"20, siguiendo en esta lógica Prats presenta cuatro campos de trabajo tales como los modelos y diseños rigurosos de enseñanza, las teorías explicativas que surgen de la investigación didáctica, la representación pedagógica, y por último "el diseño, experimentación y evaluación de estrategias didácticas y la creación de materiales y elementos que sirvan para intervenir de manera controlada en el proceso de enseñanza y aprendizaje" ${ }^{21}$ esto último - y todos los campos en particular- con un énfasis en la praxis pedagógica. Esta investigación busca trabajar en el último campo mencionado por Prats en cuanto se centra en las estrategias de enseñanza de la Historia del Derecho. El abordaje de estas temáticas presenta varios modelos de aplicación didáctica. El modelo anglosajón por ejemplo se centra en las formas en que los estudiantes aprenden determinados contenidos, con un fuerte énfasis en la psicología evolutiva. Un tipo de investigación, coherente con el modelo anglosajón y centrado en el profesor, estudiaría la forma en que el docente comprende y aprehende los nuevos avances historiográficos o del saber pedagógico y cómo esto influye en su práctica pedagógica. En este sentido, es valioso el aporte de Leinhartd el cual señala que "las explicaciones de carácter procedimental son las que más tiempo demoran en desarrollarse en los profesores principiantes quienes, en su mayoría, tienden a ofrecer a los estudiantes explicaciones basadas en contenidos disciplinares" ${ }^{22}$. Esta dificultad tensiona la posibilidad de realizar una transposición didáctica, es decir pasar de unos contenidos academicistas a unos contenidos que puedan ser

20. PRATS, Joaquín, Enseñar Historia: Notas para una didáctica renovadora. Junta de Extremadura, consejería de educación, ciencias y tecnología dirección general de ordenación, Renovación y Centros. Mérida, España. 2001. Pág. 138.
21. Ídem.
22. Henríquez - Pagés, ob. cit. Pág. 66.

Revista de Ciencias Sociales - Número 65 (2014) - Universidad de Valparáíso - ISSN 0716-7725-Valparáiso, Chile 
aprehendidos ${ }^{23}$, pues se requeriría "buscar modelos de práctica que trabajen paralelamente objetivos didácticos, preguntas sobre la naturaleza epistemológica de la historia y las ciencias sociales y, objetivos de carácter instruccional" ${ }^{24}$. Mientras el modelo anglosajón se ha centrado en cuestiones más abstractas y referidas al orden del pensamiento, el modelo Franco-Italiano se ha desarrollado en siete campos de investigación que a la vez se relacionan fuertemente por los campos destacados por Prats ${ }^{25}$.

1. Se encuentra la investigación de los objetos de enseñanza, es decir en los artefactos pedagógicos que facilitan o no determinados aprendizajes por parte de los estudiantes.

2. La investigación en los documentos de soporte de la enseñanza, tales como guías de aprendizaje, libros, y el uso que el docente le da a dichos documentos en el aula.

3. La investigación en las actividades y productos desarrollados por los estudiantes, es decir cómo estos desarrollan y enfocan su aprendizaje.

4. La investigación en situaciones particulares de enseñanza y aprendizaje, especialmente aquellas generadas de manera experimental.

5. La investigación en la construcción del saber históricogeográfico, esto es, cómo los docentes y estudiantes construyen una mentalidad histórica y geográfica.

6. Los estudios sobre la evolución histórica del currículo escolar, así como del uso y diseño de textos y manuales.

7. Por último, las investigaciones en la formación didáctica de los estudiantes de pedagogía en las universidades.

23. GÓMEZ, Miguel A., "La transposición didáctica: Historia de un concepto”, En: Revista latinoamericana de estudios educativos, Colombia, Universidad de Caldas, 2005, Nº 1, vol 1, julio / diciembre / 2005. Págs. 83-115.

24. HENRÍQUEZ - PAGÉS, ob. cit. Pág. 66.

25. Ídem, Pág. 72. Prats, ob. cit.

Facultad de Derecho y Ciencias Sociales - Universidad de Valparaíso - Chile 
En este sentido, es importante destacar el papel que juega el discurso pedagógico, entendido como toda práctica de enseñanza en la cual se trata de adecuar todos aquellos conceptos y significados de determinada disciplina, para una práctica o para una situación en la que no fueron concebidos. Se trata de convertir el conocimiento científico en conocimiento cotidiano para el docente sin que pierda su forma académica y la propia rigurosidad del conocimiento científico, en palabras de Bernstein "Es un principio que extrae (descoloca) un discurso de su práctica y contexto sustantivos y lo recoloca según su propio principio selectivo de reordenación y enfoque [...] En el proceso de descolocación y recolocación, el discurso original es sometido a una transformación que lo modifica a partir de una práctica concreta hasta otra virtual o imaginaria" ${ }^{26}$. Entonces, siguiendo a Rüsen (1987) en "The Didactics of History in West Germany: Towards a New Self-Awareness of Historical Studies" es necesario la incorporación de una didáctica que medie entre la Historia como disciplina científica y la enseñanza de la Historia. Dicho esto, uno de los problemas recurrentes en el ámbito de la enseñanza de pregrado tiene que ver con que dichas mediaciones (transposición de acuerdo a la Escuela Francesa) presentan algunas deficiencias notorias que impiden alcanzar unos correctos niveles formativos ${ }^{27}$.

Ahora bien, el proceso didáctico amparado en los puntos anteriores y en la transposición didáctica tiene una base teórica que se basa fundamentalmente en las Muestras de Desempeño Docente (MDD). El enfoque de los Estándares de las Muestras de Desempeño Docente corresponde a "expectativas de aprendizaje acerca de lo que los profesores en formación deberían saber y ser capaces de hacer, expresadas en términos de desempeños" ${ }^{28}$. Como señala

26. BERNSTEIN, Basil: La estructura del discurso pedagógico. Madrid, España. Editorial Morata. 1994.

27. RÜSEN, Jörn, "The Didactics of History in West Germany: Towards a New Self-Awareness of Historical Studies”, En: History and Theory, Vol. 26, No 3, 1987. Págs. 275-286.

28. MONTESINOS, Carmen: Muestra de desarrollo docente: Instrumentos para evaluar la calidad de la enseñanza y su impacto en el aprendizaje. Santiago, Chile. Ediciones Universidad Católica de Chile. 2009. Pág. 77.

Revista de Ciencias Sociales - Número 65 (2014) - Universidad de Valparáíso - ISSN 0716-7725-Valparáiso, Chile 
Gutiérrez $^{29}$ dentro de los elementos a tomar en consideración a la hora de evaluar los factores claves del desempeño docente se encuentra en primer lugar las aptitudes y habilidades del docente de manera global, esto es que sea un verdadero profesional de la enseñanza. El segundo factor es el uso de la planificación educativa y didáctica, es decir en qué sentido se organizan y secuencian las diferentes actividades de aprendizaje y cómo éstas en su conjunto ofrecen un cuadro de unidad respecto a los contenidos. El tercer factor a considerar son los métodos y técnicas para la enseñanza pues "se ha identificado que el proceso de aprendizaje requiere de una variedad de procedimientos, estrategias y técnicas, por ello el método brinda al profesor criterios para modelar el método que mejor responda a la situación didáctica que pone en práctica" ${ }^{30}$.

Como ya se manifestó, la investigación que se presenta tiene dos grandes referentes teóricos desde el ámbito pedagógico: el constructivismo y un modelo de aprendizaje conceptual, el cual es una estrategia de enseñanza inductiva, diseñada para ayudar a los alumnos a reforzar su comprensión de los conceptos y a practicar la examinación de hipótesis ${ }^{31}$. Este modelo sugiere además que los alumnos "construyen" su propia comprensión acerca del funcionamiento del mundo, en lugar de adquirirlo a través de formas previamente organizadas ${ }^{32}$. El modelo de aprendizaje conceptual presenta la ventaja que permite que los estudiantes trabajen la elaboración y comprobación de hipótesis usando ejemplos a partir del estudio de determinadas fuentes primarias. La implementación de este modelo requiere identificar temas, así como metas de aprendizaje claras y precisas, además de seleccionar buenos ejemplos, para secuenciarlos de tal modo que permitan desarrollar en

29. GUTIÉRREZ, Elva: "Un modelo de evaluación del desempeño docente que contribuye en la mejora de la calidad de los servicios educativos". En: Notas del Congreso Iberoamericano de Educación. Buenos Aires, Argentina. 2010.

30. Ídem. Pág. 3.

31. EGGEN - KAUCHAK, ob. cit. Pág. 148.

32. Ídem. Pág. 149.

Facultad de Derecho y Ciencias Sociales - Universidad de Valparaíso - Chile 
los alumnos habilidades de pensamiento crítico superior al trabajar el análisis de hipótesis, sugiriendo, aceptando, modificando, rechazando y finalmente identificando aquella que mejor se adecue a la información provista en los ejemplos. Este modelo normalmente no se enfoca en el aprendizaje inicial de un concepto, sino en el enriquecimiento de un concepto, es decir en la consolidación de su aprendizaje. Esta estrategia busca un aprendizaje desde la comprensión, lo que implica conectar las representaciones previas con el nuevo conocimiento. En este sentido, "el aprendizaje conceptual considera un proceso más complejo que factual, ya que dicho proceso implica una abstracción mayor de su significado esencial, identificación de características definitorias y reglas que componen el conocimiento, además de la forma como se construye el conocimiento de conceptos y explicaciones históricas y geográficas"33. Considerando por lo tanto los resultados que se presentan, las futuras sesiones debieran ser diseñadas en base a una estrategia inicial de enseñanza directa, la cual constituye una estrategia basada en la investigación sobre la eficacia del docente y caracterizada por ser ampliamente aplicable porque puede ser usada tanto para enseñar conceptos como habilidades. Básicamente, este enfoque ubica al profesor en el centro de la enseñanza, asumiendo este la responsabilidad de estructurar el contenido o la habilidad, generando explicaciones a los alumnos, dándoles oportunidades para practicar y brindándoles retroalimentación ${ }^{34}$.

33. INSTITUTO DE HISTORIA, Marco teórico de las estrategias de aprendizaje. Departamento de didáctica de la historia, Pontificia Universidad Católica de Valparaíso. Viña del Mar, Chile. 2008. Pág. 5.

34. EGGEN - KAUCHAK, ob. cit. Pág. 245.

Revista de Ciencias Sociales - Número 65 (2014) - Universidad de Valparáíso - ISSN 0716-7725-Valparáiso, Chile 
Juan Pablo Zambrano Tiznado, Andrés Sáez Geoffroy y Guillermo Zambrano Tiznado

\section{Diseño metodológico de la investigación}

\subsection{Tipo de estudio: Cuantitativa - Descriptiva}

\subsection{Variables:}

- Propuesta didáctica innovadora

- Aumento del aprendizaje

- Satisfacción de los estudiantes

Para llevar a cabo la investigación se elaboró una encuesta que se hace cargo de las tres variables en estudio. Dicho instrumento se aplicó el segundo semestre de 2012 a los alumnos de la asignatura de Historia del Derecho desarrollada el $1^{\text {er. }}$ semestre de dicho año. La encuesta se aplicó al 100\% de los estudiantes de la asignatura. Los datos fueron procesados utilizando el software SPSS y se obtuvieron los porcentajes en cada una de las posibles respuestas de acuerdo a la siguiente categorización: totalmente en desacuerdo (TD), en desacuerdo (D), indiferente (I), de acuerdo (A) o totalmente de acuerdo (TA). Tras obtener los valores respectivos se procedió con ellos a clasificarlos en función de las variables ya explicitadas. Una vez realizada la enunciación descriptiva de los resultados se establecieron diversas correlaciones en base a la proporcionalidad directa o inversa entre las diferentes variables, sea dentro de las categorías de análisis como entre diferentes preguntas y por tanto indicadores.

\section{Presentación y análisis de resultados}

\subsection{Introducción al análisis de los resultados}

Como se indicó, hemos identificado preguntas, categorizándolas en diferentes variables que el instrumento permite medir.

Para facilitar la comprensión de los resultados, estos se presentan en gráficos que acompañarán las diferentes descripciones y análisis con el fin de ilustrar los diferentes elementos y variables analizadas.

\footnotetext{
Facultad de Derecho y Ciencias Sociales - Universidad de Valparaíso - Chile
} 
Primera categoría: Propuesta pedagógica

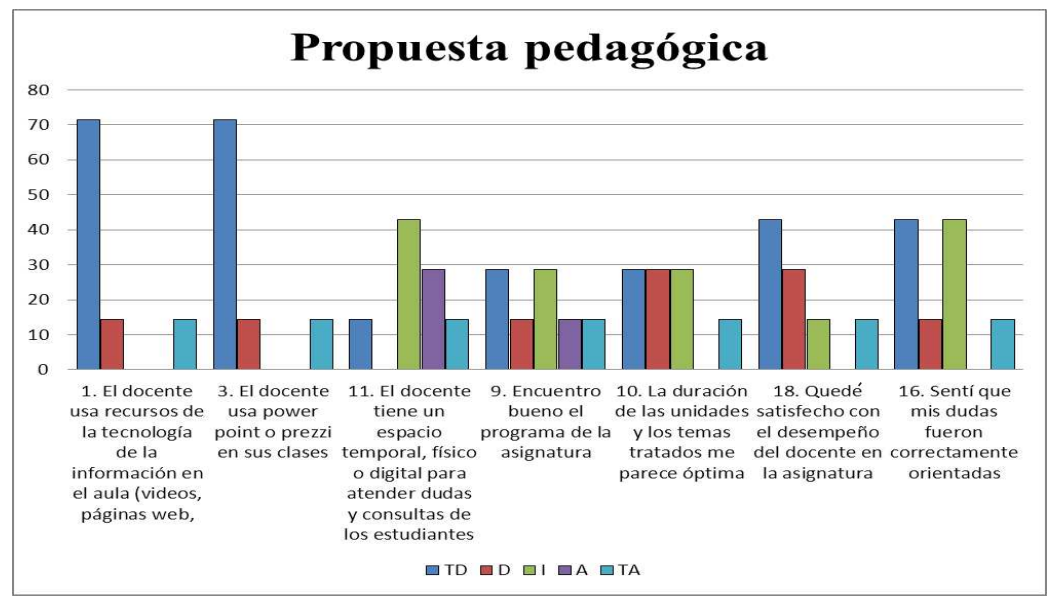

En cuanto a la variable referida al uso de elementos tecnológicos en el proceso de enseñanza-aprendizaje en la pregunta número 1, el $71,4 \%$ de los estudiantes declaró que en la impartición de la docencia de la asignatura de Historia del Derecho no se usaban recursos digitales, porcentaje que aumenta al $85 \%$ si contabilizamos los que mencionan que casi nunca se usaron ese tipo de recursos. El mismo resultado anterior se encuentra en la pregunta número 3 con los mismos porcentajes respecto al uso de recursos digitales tales como power point o prezi.

En cuanto al espacio para atender dudas, a los estudiantes les resultó indiferente la atención o la existencia de un espacio donde poder atender y ubicar al docente con un 42,9\%. Dentro de la misma temática, los estudiantes consideran que sus dudas fueron muy poco atendidas con un totalmente en desacuerdo de un $42,9 \%$, en desacuerdo $14,3 \%$ o indiferente 28,6\%. Algo similar se repite en cuanto al programa de la asignatura donde las preguntas 9 y 10 tienen de todas las preferencias y sus resultados son estables. 
Segunda categoría: Propuesta pedagógica

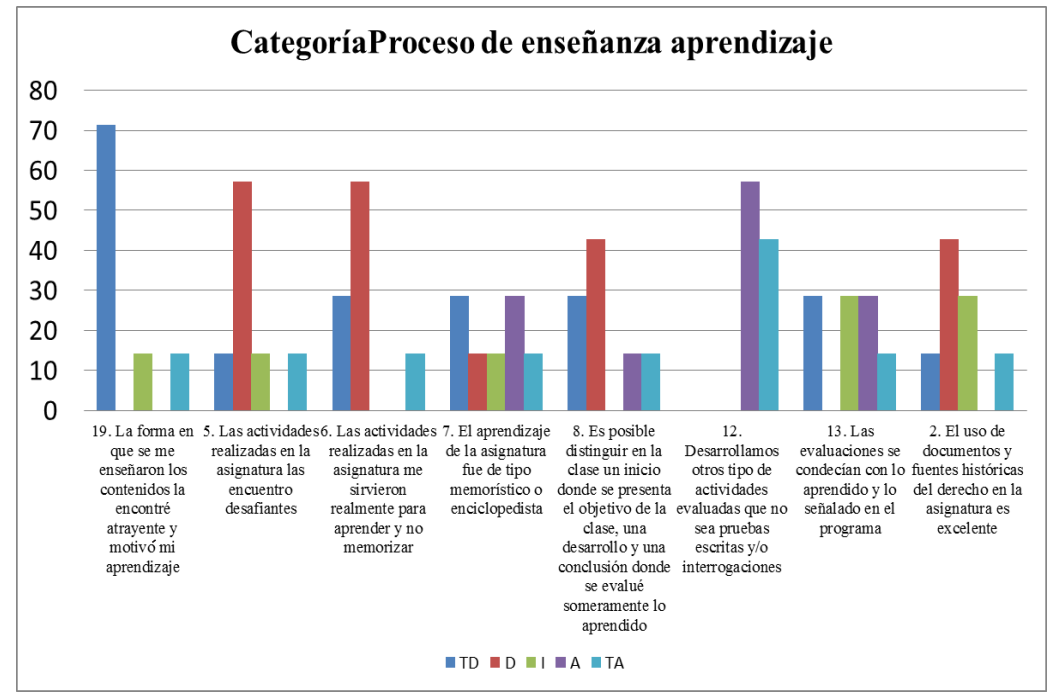

En cuanto a la forma de enseñanza y el proceso de aprendizaje, la gran mayoría de los encuestados con un $71,4 \%$ señalaron que la metodología en la cual se les enseñaba resulta poco atrayente y que no motivaba el proceso de aprendizaje. En dicha línea, las actividades realizadas en la asignatura de acuerdo a los resultados en primer lugar fueron poco desafiantes (pregunta 5) con un $71,4 \%$, en tanto declararon mayoritariamente que las mismas actividades favorecieron la memorización con un 85,7\% (Pregunta 6). Por otra parte, en la pregunta 2 el 59,2\% declaró que no se usó de manera constante o simplemente no se usaron documentos históricos.

Estos resultados que bien pudieran tener una continuidad se diluyen en la pregunta 7 porque si bien el 42,9\% declaró que la asignatura favorecía la memorización antes que el aprendizaje, en tanto el otro $42,9 \%$ señaló que no fue memorística. Existe consenso en los encuestados que se desarrollaron actividades evaluadas más allá de las pruebas sea en grado intermedio $(57,1 \%)$ o máximo (42,9\%). En este sentido las evaluaciones en cuanto a si estaban conformes con los objetivos presenta porcentajes en todas las opciones.

Facultad de Derecho y Ciencias Sociales - Universidad de Valparaíso - Chile 


\section{Tercera Categoría: Satisfacción estudiantil}

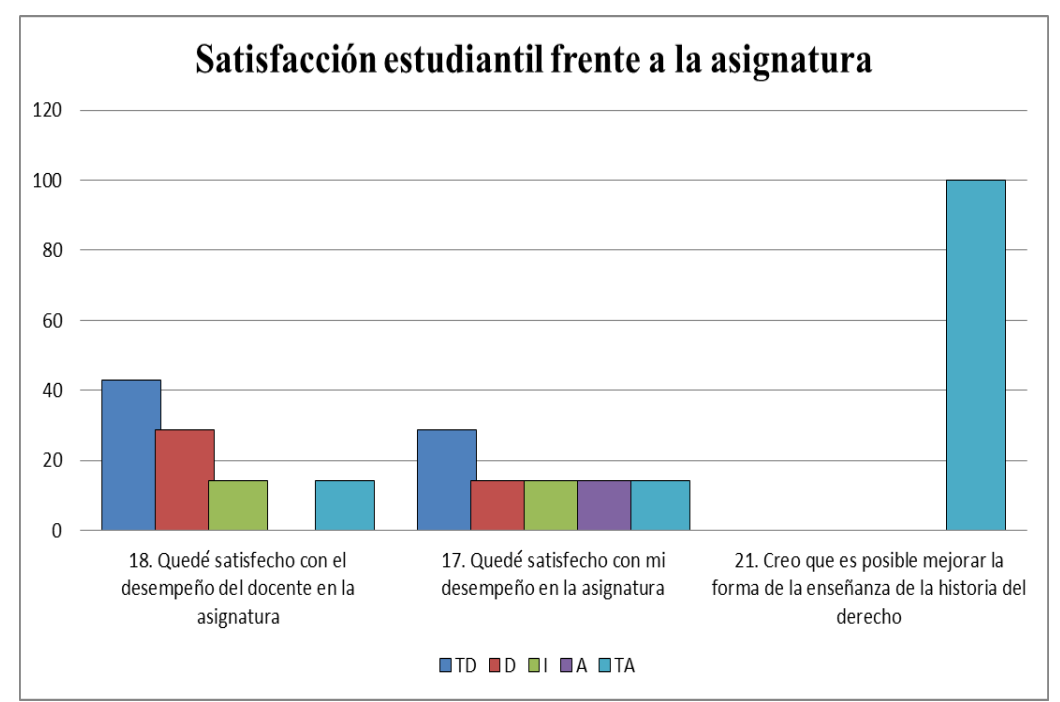

En cuanto al grado de conformidad de los estudiantes con la asignatura, los resultados negativos alcanzan en conjunto un $71,5 \%$ de desaprobación, un 14,3 es indiferente y otro 14,3 se manifestó a favor de su desempeño. En cuanto al desempeño personal se mantienen todas las variables con resultados similares.

Respecto a la posibilidad de mejorar la enseñanza, todos, el 100\% se declaró a favor del mejoramiento de todas las prácticas posibles dentro del proceso de enseñanza-aprendizaje.

\subsection{Análisis de datos}

Para el análisis de datos se utilizó la correlación de Pearson para medir dos variables que se pudieran extraer de acuerdo a los puntajes. Las correlaciones más significativas fueron: 
Juan Pablo Zambrano Tiznado, Andrés Sáez Geoffroy y Guillermo Zambrano Tiznado

\begin{tabular}{|c|c|}
\hline Coef. & Variables relacionadas (Correlación Pearson) \\
\hline \multirow[t]{2}{*}{1,0} & $\begin{array}{l}\text { 1. El docente usa recursos de la tecnología de la información en } \\
\text { el aula (videos, páginas web, etc.). }\end{array}$ \\
\hline & 3. El docente usa power point o prezi en sus clases. \\
\hline
\end{tabular}

\begin{tabular}{|c|l|}
\hline \multirow{2}{*}{0,2} & $\begin{array}{l}\text { 2. El uso de documentos y fuentes históricas en la asignatura es } \\
\text { óptimo. }\end{array}$ \\
\cline { 2 - 3 } & $\begin{array}{l}\text { 4. En las clases de Historia del Derecho el rol de los estudiantes } \\
\text { es activo. }\end{array}$ \\
\hline
\end{tabular}

\begin{tabular}{|l|l|}
\hline \multirow{2}{*}{0,9} & $\begin{array}{l}\text { 5. Las actividades realizadas en la asignatura las encuentro } \\
\text { desafiantes. }\end{array}$ \\
\cline { 2 - 3 } & $\begin{array}{l}\text { 2. El uso de documentos y fuentes históricas del derecho en la } \\
\text { asignatura es óptimo. }\end{array}$ \\
\hline
\end{tabular}

\begin{tabular}{|c|l|}
\hline \multirow{2}{*}{0,9} & $\begin{array}{l}\text { 5. Las actividades realizadas en la asignatura las encuentro } \\
\text { desafiantes. }\end{array}$ \\
\cline { 2 - 2 } & $\begin{array}{l}\text { 6. Las actividades realizadas en la asignatura me sirvieron } \\
\text { realmente para aprender y no memorizar. }\end{array}$ \\
\hline
\end{tabular}

\begin{tabular}{|c|c|}
\hline \multirow{2}{*}{0,3} & \begin{tabular}{l} 
9. Encuentro bueno el programa de la asignatura. \\
\cline { 2 - 2 }
\end{tabular} \\
$\begin{array}{c}\text { 10. La duración de las unidades y los temas tratados me parece } \\
\text { óptima. }\end{array}$ \\
\hline
\end{tabular}

\begin{tabular}{|l|l|}
\hline \multirow{3}{*}{$-0,6$} & $\begin{array}{l}\text { 12. Desarrollamos otros tipo de actividades evaluadas que no sea } \\
\text { pruebas escritas y/o interrogaciones. }\end{array}$ \\
\cline { 2 - 3 } & $\begin{array}{l}\text { 4. En las clases de Historia del Derecho el rol de los estudiantes } \\
\text { es activo. }\end{array}$ \\
\hline
\end{tabular}

\begin{tabular}{|c|c|}
\hline \multirow{3}{*}{$-0,5$} & $\begin{array}{l}\text { 22. Me gustaría tener más participación en la construcción del } \\
\text { conocimiento generado en la asignatura. }\end{array}$ \\
\cline { 2 - 3 } & $\begin{array}{l}\text { 2. El uso de documentos y fuentes históricas del derecho en la } \\
\text { asignatura es óptimo. }\end{array}$ \\
\hline
\end{tabular}

En primer lugar se pudo establecer una correlación perfecta entre el uso de elementos tecnológicos para el apoyo pedagógico y la utilización de power point o prezi. La correlación positiva perfecta (es decir con valor 1) demuestra que los estudiantes relacionan el uso de tecnología con dichos programas, lo que constituye una simpli- ficación de los recursos tecnológicos que se pueden usar en el aula. El uso de una plataforma, de juegos digitales u otros elementos de aprendizaje

Facultad de Derecho y Ciencias Sociales - Universidad de Valparaíso - Chile 
digital o tecnológico es más amplio que el solo uso del recurso para la proyección; no basta prezi o power point porque lo relevante es el uso que el docente le puede dar a power point o a herramientas de ese tipo.

Una relación menor se da cuando analizamos las variables de uso de fuentes históricas y el rol de los estudiantes. La correlación baja de 0,2 muestra que hay un factor que influye negativamente en el aumento de dicho factor. En este sentido, el 42,9\% se mostró indiferente frente al carácter activo de su rol como estudiante. Así mismo, el uso de una determinada técnica pedagógica es función del docente quien evaluando el contexto de la enseñanza decide de mottu propio la utilización de una estrategia didáctica diferente. De hecho, si correlacionamos la variable uso de documentos históricos con la variable significancia de los contenidos de aprendizaje, nos da una correlación de 0,5 lo que indica y reafirma lo anteriormente enunciado. En este mismo análisis de variables, encontramos un valor de 0,9 cuando se relacionan las variables de actividades desafiantes con el uso de documentos, es decir, los estudiantes piden el uso de este tipo de técnicas con el fin de encontrar y mejorar el desarrollo de su propio quehacer estudiantil, lo que reafirma el indicador anterior.

Los mismos estudiantes creen que la realización de actividades desafiantes permite mejores aprendizajes con una correlación de 0,9 ; sin embargo, en el caso del curso de Historia del Derecho el 71,4\% encontró las actividades poco desafiantes así como el 85,7\% tampoco encontró un aprendizaje constructivista, sino que de tipo memorístico y enciclopédico, lo que se subsana con alguna propuesta de tipo pedagógica que involucre una mayor dinámica de actividades. En este sentido, la correlación entre el programa de la asignatura, su duración y la disposición de las unidades de aprendizaje de los contenidos de Historia del Derecho, es baja con un 0,3. Esta diversidad de opiniones permite reafirmar que es un elemento privativo del docente poder adaptar el programa de la asignatura determinando y adaptando los énfasis de los contenidos, actividades y acciones a las necesidades de sus estudiantes; es en esa libertad de cátedra donde se enmarcaría una propuesta didáctica innovadora.

Se produce una correlación en sentido opuesto con un -0,6 cuando tomamos las variables de rol activo de los estudiantes y otro tipo de actividades, es decir, los estudiantes sienten indiferencia frente a su rol

Revista de Ciencias Sociales - Número 65 (2014) - Universidad de Valparaíso - ISSN 0716-7725-Valparaíso, Chile 
$(42,9 \%)$ aun cuando se sienten favorecidos cuando se entregan diferentes formas de evaluación y trabajo lo que reafirma la argumentación anterior. $\mathrm{Al}$ respecto, se produce una correlación inversa de $-0,5$ cuando se trata de documentos históricos, lo que reafirma que no se realizaron actividades que favorecieran su uso.

Respecto al uso de algún tipo de plataforma y acceso a bibliografía, se produce una correlación negativa casi perfecta con -0,7 señalándose que aunque no se utilizó la plataforma de forma activa, los estudiantes sí tuvieron acceso a la bibliografía. Lo mismo ocurre en el caso de la correlación negativa de $-0,8$ entre uso de plataforma y recursos tecnológicos con uso de TIC's en el aula, es decir, el estudiante establece la relación aunque no se hayan utilizado dichos recursos en concreto.

Otro tipo de correlación es la que se establece entre las variables de significación y participación de los estudiantes que tiene un resultado de -0,4. Es decir, mientras por una parte los estudiantes manifiestan que no le son significativos los contenidos, si desean tener más participación en la construcción de su propio conocimiento. Resulta interesante que la construcción de conocimiento se asocie directamente a la participación y motivación que logre realizar el docente con sus estudiantes. A raíz de estos mismos factores, la correlación entre contenido y formas de enseñanza es de - 0,1 , y enseñanza y participación es de $-0,3$, resultado que se repite en cuanto a posibilidades de mejora y participación.

Por último, dos correlaciones que refuerzan la idea de necesidad de una propuesta pedagógica es el resultado de $-0,5$ que existe entre la mejor participación y deseo de participar en la construcción del conocimiento y el uso de una técnica específica como sería el uso de fuentes y documentos históricos. En este sentido, los mismos estudiantes que encuentran posible mejorar la docencia y el proceso de enseñanzaaprendizaje señalan que la forma en que se está ejerciendo la práctica docente $(-0,5)$ no da cuenta de estas posibilidades y que requiere un cambio estructural.

Facultad de Derecho y Ciencias Sociales - Universidad de Valparaíso - Chile 


\section{Conclusiones}

Como se indicó en la introducción, este trabajo tiene una finalidad diagnóstica y descriptiva. Su objetivo era entregar información sobre las correlaciones entre las variables en estudio para determinar la necesidad de implementar un nuevo diseño didáctico.

Dentro de los objetivos generales, se encontraba "identificar las falencias que presenta en el aprendizaje de los estudiantes el uso de una metodología tradicional de enseñanza en la asignatura Historia del Derecho". Dentro de dichas falencias se han detectado dificultades en cuanto a una implementación tecnológica (uso de power point u otras herramientas de proyección de información), así como la necesidad de implementar técnicas, entre ellas el trabajo de la heurística y hermenéutica histórica, exégesis, cartografía histórica o lecturas adecuadas a los contenidos.

Dentro de los mismos objetivos se encontraba "determinar las expectativas que los alumnos manifiestan respecto a un cambio en la didáctica de la asignatura". En este ámbito, se evidenció una baja satisfacción del estudiante. Este resultado puede reflejar la aparente inutilidad de la asignatura para el estudiante, el bajo nivel de dominio de los contenidos por parte del docente (por no ser un historiador profesional) y el rol pasivo asignado al estudiante en el proceso de aprendizaje (por las bajas competencias pedagógicas del docente).

El resultado del estudio es que existe la necesidad de planificar, a partir de las expectativas de los estudiantes y la literatura especializada en Didáctica de la Historia, una estrategia para estudiar Historia del Derecho, cuestión que se transforma en un compromiso ineludible a partir del diagnóstico y los resultados arrojados por este, especialmente para mejorar los indicadores relativos a la satisfacción personal hacia el docente y la asignatura. Sin embargo, la posibilidad real de responder a estas necesidades se ven impedidas por problemas estructurales de la educación legal en Chile. En efecto, existiendo una interesante bibliografía sobre didáctica de la historia no ocurre lo mismo con la Historia del Derecho o con las Ciencias Jurídicas en general y aunque existiera, es el docente el que debe tener la formación no sólo disciplinar sino pedagógica que le permita convertirse en un académico crítico de sus propias prácticas.

Revista de Ciencias Sociales - Número 65 (2014) - Universidad de Valparáíso - ISSN 0716-7725-Valparaíso, Chile 


\section{BIBLIOGRAFÍA}

COLOMA, Rodrigo: "El ocaso del profesor Binns. Un ensayo acerca de la enseñanza del derecho en Chile". En: Ius et Praxis. Talca, Chile. 2005, vol.11. $\mathrm{N}^{\circ} 1$.

AGÜERO, Claudio y ZAMBRANO, Juan Pablo: "El derecho de acceso a la información como garantía de una educación de calidad". En: Revista de Ciencias Sociales, Universidad de Valparaíso, Facultad de Derecho. Valparaíso, Chile. 2009.

SOLARI, Enzo: "El currículo chileno de estudios jurídicos". En: Revista de Derecho de la Pontificia Universidad Católica de Valparaíso. Valparaíso, Chile 2012.

BRUNNER, José Joaquín: "Globalización y el futuro de la educación: tendencias, desafíos y estrategias". En: Seminario sobre prospectiva de la educación en la región de América Latina y el Caribe, UNESCO Santiago, Chile. 23 al 25 de agosto del 2000.

DELORS, Jaques: La educación encierra un tesoro, Editorial Santillana, UNESCO, 1996.

DÍAZ, Frida y HERNÁDEZ, Gerardo: Estrategias Docentes para un Aprendizaje Significativo: una interpretación constructivista.

México: Mc Graw Hill, 2002. p.143, Vid. EGGEN, Paul D. -

KAUCHAK, Donald P.: Estrategias docentes: Enseñanza de contenidos curriculares y desarrollo de habilidades de pensamiento. D.F, México. Editorial Fondo de cultura económica, 2005.

HABERMAS, Jürgen: Ciencia y técnica como ideología. Madrid, España. Editorial Tecnos, 1986.

GRUNDY, Shirley: Producto o praxis del currículum, Madrid, España. Editorial Morata, 1999.

PIAGET, Jean, Psicología y pedagogía. Barcelona, España. Editorial Ariel, 2001.

PÉREZ CABANÍ, María: "La formación del profesorado para enseñar estrategias de aprendizaje". En: Moreno, Carlos, Estrategias de enseñanza y aprendizaje: formación del profesorado y aplicación en la escuela. Editorial Visor, 2002.

COOL, César: "Aprendizaje por descubrimiento frente a aprendizaje por recepción”. En: Juan García Madruga. Desarrollo psicológico y educación. Madrid, España. Editorial Alianza. 1999.

Facultad de Derecho y Ciencias Sociales - Universidad de Valparaíso - Chile 
RODRÍGUEZ, Luz: La teoría del aprendizaje significativo. Tenerife, España. Centro de educación a distancia, 2008.

GOOG, Thomas y BROPHY, Jere E.: Psicología educativa contemporánea. D.F., México. Editorial McGraw Hill, 2000.

HENRÍQUEZ Rodrigo y PAGÉS, Joan: "La investigación en didáctica de la historia". En: Educación XXI Revista de la Facultad de Educación de la Universidad Autónoma de Barcelona, España. 2004, No 7, pp. 63-84.

PAGÉS, Joan, "Enseñar a enseñar Historia: la formación didáctica de los futuros profesores de Historia”. En: Llopis, Miguel. Miradas a la historia. Murcia: Universidad de Murcia. 2004.

PRATS, Joaquín, Enseñar Historia: Notas para una didáctica renovadora. Junta de Extremadura, consejería de educación, ciencias y tecnología dirección general de ordenación, Renovación y Centros. Mérida, España. 2001.

GÓMEZ, Miguel A., "La transposición didáctica: Historia de un concepto", En: Revista latinoamericana de estudios educativos, Colombia, Universidad de Caldas, 2005, No 1, vol 1, julio / diciembre / 2005, pp. 83-115.

BERNSTEIN, Basil: La estructura del discurso pedagógico. Madrid, España. Editorial Morata. 1994.

RÜSEN, Jörn, “The Didactics of History in West Germany: Towards a New Self-Awareness of Historical Studies”, En: History and Theory, Vol. 26, No 3 , 1987, pp. 275-286.

MONTESINOS, Carmen: Muestra de desarrollo docente: Instrumentos para evaluar la calidad de la enseñanza y su impacto en el aprendizaje. Santiago, Chile. Ediciones Universidad Católica de Chile. 2009.

GUTIÉRREZ, Elva: “Un modelo de evaluación del desempeño docente que contribuye en la mejora de la calidad de los servicios educativos". En: Notas del Congreso Iberoamericano de Educación. Buenos Aires, Argentina. 2010.

INSTITUTO DE HISTORIA, Marco teórico de las estrategias de aprendizaje. Departamento de didáctica de la historia, Pontificia Universidad Católica de Valparaíso. Viña del Mar, Chile. 2008. p. 5. 